\title{
Kounis Syndrome: \\ Dexketoprofen-Associated ST-Elevation Myocardial Infarction
}

\author{
Aytekin Aksakal ${ }^{1}$, Zeki Şimşek², Diyar Köprülü ${ }^{1}$, Uğur Arslan $^{3}$ \\ ${ }^{1}$ Cardiology Department, Ordu State Hospital, Ordu, Turkey \\ ${ }^{2}$ Cardiology Department, Kartal Koşuyolu Training and Education Hospital, Istanbul, Turkey \\ ${ }^{3}$ Cardiology Department, Samsun Training and Education Hospital, Samsun, Turkey
}

\section{Doi: 10.12890/2021_003006 - European Journal of Case Reports in Internal Medicine - ๑ EFIM 2021}

Received: 15/10/2021

Accepted: 24/10/2021

Published: $12 / 11 / 2021$

How to cite this article: Aksakal A, Şimşek Z, Köprülü D, Arslan U. Kounis Syndrome: Dexketoprofen-associated ST-elevation myocardial infarction. EJCRIM 2021;8: doi:10.12890/2021_003006.

Conflicts of Interests: The authors declare there are no competing interests.

This article is licensed under a Commons Attribution Non-Commercial 4.0 License

\section{ABSTRACT}

Allergic angina and allergic myocardial infarction are common diseases associated with acute coronary syndromes and encompass a wide spectrum of mast cell activation disorders termed "Kounis Syndrome". We present here a patient with Kounis syndrome presenting with sudden cardiac arrest after intravenous infusion of dexketoprofen in the emergency room.

\section{LEARNING POINTS}

- $\quad$ There are 3 types of Kounis syndrome, of which Type I, allergic coronary vasospasm, is the most common.

- The most common presentation of Kounis syndrome is ST-elevation myocardial infarction.

- Although not all cases of Kounis syndrome are reported, they are common in clinical practice and many more causal factors are predicted to play a role in the future.

\section{KEYWORDS}

Kounis syndrome, dexketoprofen, allergic myocardial infarction

\section{INTRODUCTION}

Kounis syndrome has been established as a hypersensitivity coronary disorder caused by various factors including drugs, environmental conditions, foods, and coronary stents. Allergic, hypersensitivity, anaphylactic and anaphylactoid reactions are associated with this syndrome. Kounis syndrome is not a rare disease, it appears to be an infrequently diagnosed clinical entity. Patients present the same mediators released from the same inflammatory cells than in acute coronary events of non-allergic etiology. Intravenous (iv) dexketoprofen is a non-steroidal anti-inflammatory drug (NSAID) used to treat many conditions in emergency services such as toothache, muscle pain, and fractures. There is evidence however showing that increasing use around the world of non-steroidal anti-inflammatory drugs is causing an increased incidence of acute myocardial infarction ${ }^{[1]}$.

\section{CASE DESCRIPTION}

A 49-year-old male patient was admitted to hospital with diffuse muscle pain. Intravenous dexketoprofen infusion was started in the emergency room due as the patient complained of widespread muscle pain. After the start of the infusion, the patient suddenly developped hypotension and chest pain. The patient was sent to us, from the 1st level hospital to the city hospital, after the first ECG (Fig. 1) showed 
diffuse ST-elevation. Despite intravenous isotonic and subcutaneous epinephrine administration in the emergency department, the patient developed sudden cardiac arrest. The patient underwent cardiopulmonary resuscitation for 30 minutes. In the laboratory results of the patient in the emergency room, an increase in cardiac enzymes was detected. The laboratory results indicated: Mass Ck-Mb: $8.59 \mathrm{ng} / \mathrm{ml}$, Troponin I: $2.59 \mathrm{ng} / \mathrm{dl}$. Arterial blood gas results: $\mathrm{pH}: 7.18, \mathrm{PCO}_{2}: 61.2 \mathrm{mmHg}, \mathrm{PO}_{2}: 16.6 \mathrm{mmHg}$

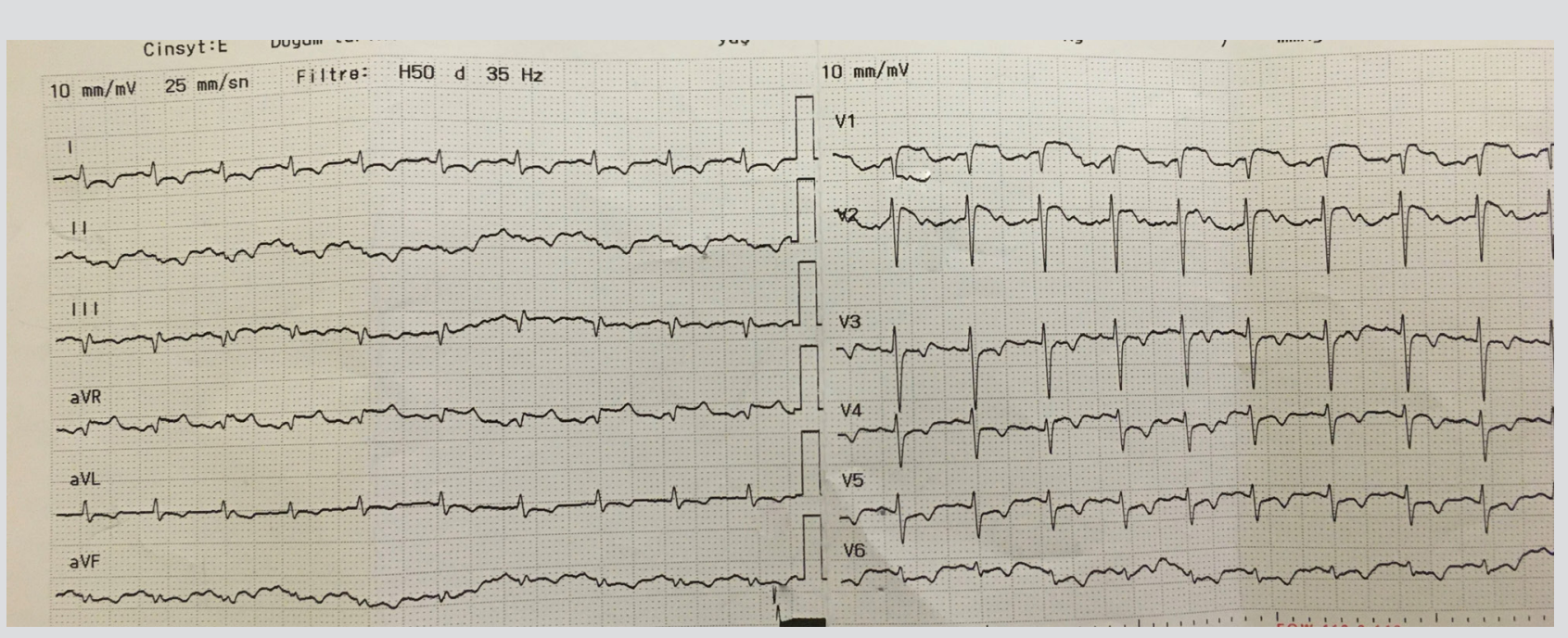

Figure 1: ECG showing ST elevation in Avr and common ST depression other precordial derivations (anterior wall myocardial infarction)

The patient was urgently taken to the catheter laboratory for coronary angiography. Normal coronary arteries were detected by coronary angiography. The patient's blood pressure did not rise despite intravenous norepinephrine and isotonic infusions, and he died despite receiving corticosteroid and low molecular weight heparin in the intensive care unit. Verbal consent was obtained from the patient's relatives for the presentation of this case.

\section{DISCUSSION}

Kounis syndrome is defined as the association of acute coronary syndromes, including coronary spasm, acute myocardial infarction, and stent thrombosis, with conditions associated with mast cell and platelet activation. It involves interrelated and interacting inflammatory cells such as macrophages and T-lymphocytes. This syndrome is caused by inflammatory mediators such as histamine, platelet activating factor, arachidonic acid products, neutral proteases, and various cytokines and chemokines released during the allergic activation process ${ }^{[2]}$. Various causes have been found to trigger Kounis syndrome, and its incidence is increasing rapidly. Among the 51 cases of Kounis syndrome reported to the International Pharmacovigilance Agency (VigiBase ${ }^{\mathrm{TM}}$ ) during 2010-2014, almost half the cases (22 reports) were from 2014. Most cases occurred in the USA, and non-steroidal anti-inflammatory drugs were the most common trigger drugs ${ }^{[3]}$. Although it is not a rare disease, it is rarely diagnosed and easily overlooked ${ }^{[4]}$.

Three variants of Kounis syndrome have been described ${ }^{[5]}$. Type I variant (coronary spasm) appears to represent a manifestation of endothelial dysfunction or microvascular angina; cases involve patients with normal or near-normal coronary arteries without predisposing factors for coronary artery disease. Type II variant includes patients with atheromatous disease in which acute release of inflammatory mediators can induce coronary artery spasm with normal cardiac enzymes and troponins, or coronary artery spasm with plaque erosion or acute myocardial rupture. Type III variant is diagnosed in patients who had stent implantation and died suddenly.

The therapeutic management of Kounis syndrome is a challenging procedure because it must simultaneously treat both cardiac and allergic symptoms. Medications given to treat cardiac symptoms can exacerbate allergies, and medications given to treat allergic symptoms can worsen heart function ${ }^{[6]}$. In patients with Type I variant, treatment of the allergic event alone may relieve symptoms. Intravenous corticosteroids such as hydrocortisone at a dose of 1-2 mg/kg/day, $\mathrm{H} 1$ and $\mathrm{H} 2$ antihistamines such as diphenhydramine at a dose of 1-2 mg/ $\mathrm{kg}$ and ranitidine at a dose of $1 \mathrm{mg} / \mathrm{kg}$ are sufficient. In patients with type II variant, treatment should be initiated with an acute coronary event protocol with corticosteroids and antihistamines. The use of $\beta$-blockers may exaggerate coronary spasm due to the intolerable effect of $\alpha$-adrenergic receptors. In patients with type III variant, it is recommended to follow an acute myocardial infarction protocol with 


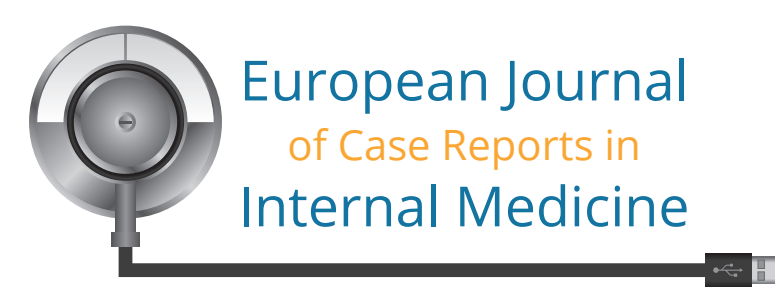

emergency aspiration of intrastent thrombus ${ }^{[6]}$. Corticosteroids and antihistamines can be used in patients with ongoing symptoms. The last option is surgical removal of the stent ${ }^{[7]}$.

Dexketoprofen trometamol is a modified, non-selective COX inhibitor with a rapid onset of action, available in both oral and parenteral formulations. It is used in the symptomatic treatment of mild to moderate pains such as musculoskeletal pain, dysmenorrhea, toothache, postoperative pain.

\section{CONCLUSION}

To our knowledge, this is the first case of Kounis caused by dexketoprofen reported in the literature. Physicians should be prepared for these rare events that can lead to serious complications.

\section{REFERENCES}

1. Bally M, Beauchamp M-E, Abrahamowicz M, Nadeau L, Brophy JM. Risk of acute myocardial infarction with real-world NSAIDs depends on dose and timing of exposure. Pharmacoepidemiol Drug Saf 2017:27: 69-77.

2. Kounis NG. Coronary hypersensitivity disorder: Kounis syndrome. Clin Ther 2013;35: 563-71.

3. Renda F, Landoni G, Trotta F, Piras D, Finco G, Felicetti P, et al. Kounis Syndrome: an analysis of spontaneous reports from the international pharmacovigilance database. International J Cardiol 2016;203: 217-20.

4. Kounis NG, Giannopoulos S, Soufras GD, Kounis GN, Goudevenos J. Foods, drugs, and environmental factors: new Kounis syndrome culprits. Intern Med 2015;54: 1577-82.

5. Tsigkas G, Chouchoulis K, Kounis NG, Alexopoulos D. The allergic reaction reveals a non-fatal late stent thrombosis. A new subtype of Kounis syndrome? International J Cardiol 2011; 149:281-2

6. Agile C, Nugent K, Shome GP, Kounis NG. Treatment of Kounis syndrome. International J Cardiol 2010; 143:223-6.

7. Atoui R, Mohammadi S, Shum-Tim D. Surgical removal of occluded stents: when stenting is a problem. Interact Cardiovasc Thorac Surg 2009;9:736-8. 